\title{
Mobility activation in thermally deposited CdSe thin films
}

\author{
KANGKAN SARMAH* and RANJAN SARMA \\ Department of Physics, Gauhati University, Guwahati 781 014, India
}

MS received 19 May 2008; revised 26 April 2009

\begin{abstract}
Effect of illumination on mobility has been studied from the photocurrent decay characteristics of thermally evaporated $\mathrm{CdSe}$ thin films deposited on suitably cleaned glass substrate held at elevated substrate temperatures. The study indicates that the mobilities of the carriers of different trap levels are activated due to the energy of incident illumination, which results in the existence of two distinct trap levels. In each trap depth the energy of the trap increases linearly. It infers that there is a linear distribution of traps of different energies below the conduction band.
\end{abstract}

Keywords. Cadmium selenide thin film; trap depth; photocurrent decay.

\section{Introduction}

CdSe is a binary semiconductor and among the II-VI group of semiconductor compounds, it is considered as an important material for the development of different optoelectronic devices (Sathyalatha et al 1989; Baban et al 2000; Al-Ani et al 2002) because of its high photosensitive nature and suitable intrinsic bandgap (Shreekanthan et al 2003). In recent years special attention has been given to the investigation of photoelectronic properties of CdSe thin films in order to improve the performances of devices and also for finding new applications (Pal et al 1993; Oduor and Gould 1995). Different growing methods for preparation of $\mathrm{CdSe}$ thin films have been reported (Baban et al 2005; Kale and Lokhande 2005). Physical vapour deposition method is often used because it offers many possibilities to modify the deposition parameters and to obtain films with determined structure and properties. Irrespective of preparation conditions such deposited films are associated with different types of native and foreign imperfections which greatly influence the optoelectronic properties of the films. Native defects such as various traps having energy in the range $0 \cdot 15-0.8 \mathrm{eV}$ (Kindleysides and Woods 1970; Kalita et al 1999) can cause considerable change in the electrical and optical properties of semiconductor thin films. These defects characterize the electronic properties of the materials, because they give rise to charge centre acting as donors and acceptors (Serpi 1976). In view of the lack of information concerning the effect of illumination on trapping spectrum in thermally evaporated CdSe thin films, in this paper an assessment has been made regarding the influ-

*Author for correspondence (ks_guphys@rediffmail.com) ence of illumination on mobility activation process as well as on the evaluation of trap depths at different ambient conditions.

\section{Experimental}

Thin films of CdSe were deposited at elevated substrate temperatures $\left(T_{\mathrm{s}}\right)$, on chemically and ultrasonically cleaned glass substrates at a vacuum of the order of $10^{-6}$ torr. Pure (99.999\%) bulk CdSe sample was used as the source material. Prior to deposition of the thin films, high purity aluminium electrodes were vacuum evaporated on glass substrates on which the films were then deposited to obtain a gap type cell configuration of $10 \times 7 \mathrm{~mm}$ geometry. Thin tantalum boats of proper size and shape were used as the source heater. A suitably designed and assembled multiple beam interferometer was used to measure the thickness $\left(t_{\mathrm{t}}\right)$ of the films with an accuracy of $\pm 15 \AA$. An ECIL electrometer amplifier of input impedance of $10^{14} \Omega$ (and higher) was used to measure dark and photocurrents. To apply bias, a series of highly stable dry cells of emf $9 \mathrm{~V}$ each were used. The sample was kept suspended by thin enameled copper wires inside a continuously evacuated glass jacket. The entire experimental set up including the observer was housed in a suitably fabricated Faraday cage in order to avoid pick-up noises. For white light illumination tungsten halogen projector lamp of 250 watt, operating at maximum voltage $24 \mathrm{~V}$, was used and the intensities were measured by using a highly sensitive APLAB luxmeter. Electrical heater was used to increase the temperature $(T)$ of the thin film sample from room temperature to any desired level. The measurement of temperature was done with the help of copper constantan thermocouple and a digital microvoltmeter arrangement. 


\section{Mobility activation in CdSe thin films}

The trap depths were calculated by using the following simple decay law

$$
I_{\mathrm{t}}=I_{\mathrm{o}} \exp (-p t),
$$

where $p$ is the probability of escape of an electron from the trap per second and is given by (Randall and Wilkins 1945)

$$
p=S \exp (-E / k T),
$$

where $E$ is the trap depth for electrons below the bottom of the conduction band or top of the valanced band, $k$ the Boltzmann constant, $T$ the ambient temperature in $K, I_{0}$ the photocurrent at the termination of illumination, $I_{\mathrm{t}}$ the photocurrent at any subsequent time, $t$, after the termination of illumination and $S$ the frequency factor defined in terms of number per second that the quanta from the lattice vibrations (phonons) attempt to eject the electron from the trap, multiplied by the probability of transition of the ejected electron to the conduction band (Srivastava and Singh 1970).

From the two relations (1) and (2), the expression for trap depth is given by

$$
E=k T\left[\ln S-\ln \left\{\ln \left(I_{\mathrm{d}} / I_{\mathrm{t}}\right) / t\right\}\right] .
$$

The probability of an electron escaping from a trap of depth $E$ and cross-section for its capture $S_{\mathrm{t}}$, at a temperature $T$, is given by (Bube 1960)

$$
p=N_{\text {eff }} v_{\text {th }} S_{\mathrm{t}} \exp (-E / k T),
$$

where $N_{\text {eff }}$ is the effective density of states in the conduction band and $v_{\text {th }}$ the thermal velocity of the electrons. Here the product $N_{\text {eff }} v_{\text {th }} S_{\mathrm{t}}$ represents the frequency factor, $S$.

For finding $S$, the values of these three parameters $N_{\text {eff, }}$, $v_{\text {th }}$ and $S_{\mathrm{t}}$ were calculated separately. $N_{\text {eff }}$ was calculated from the conductivity data by using the relation, $n=\sigma / e \mu$ (Bube 1967) where $\sigma$ is the conductivity evaluated from the experimental data of the photocurrent, $I_{0}$. In this case, it is assumed that at comparatively low temperatures, the number of occupied energy levels in the conduction band i.e. $n$, is identical with $N_{\text {eff. }} \mu$ is the mobility of the electrons in CdSe sample whose value was taken as $580 \mathrm{~cm}^{2} \mathrm{~V}^{-1} \mathrm{~s}^{-1}$ (Devlin 1967). The thermal velocity, $v_{\text {th }}$, of an electron was calculated at different ambient temperatures by using the relation,

$$
v_{\text {th }}=\left(2 k T / m^{*}\right)^{1 / 2},
$$

where $m^{*}$ is the effective mass of an electron which was taken to be $0.13 \mathrm{~m}_{\mathrm{e}}$ (Devlin 1967). The capture crosssection is given by

$$
S_{\mathrm{t}}=\pi r^{2},
$$

where $r$ is the radius of the capture centre. It was evaluated by putting Coulomb energy of interaction of an electron with the corresponding trap equal to the thermal energy of the electron at temperature, $T$ (Bube 1960) i.e.

$$
e^{2} / r \varepsilon=k T,
$$

where $\varepsilon$ is the dielectric constant of CdSe sample whose value is taken to be 5.76 (Kalita et al 2003). Using (7) in (6) the relation for capture cross section is given by

$$
S_{\mathrm{t}}=\pi e^{4} / k^{2} T^{2} \varepsilon^{2} .
$$

At $300 \mathrm{~K}$,

\begin{tabular}{|c|c|c|c|c|c|c|}
\hline \multirow{2}{*}{$\begin{array}{l}\text { Ambient } \\
\text { temperature }(\mathrm{K})\end{array}$} & \multirow{2}{*}{$\begin{array}{c}\text { Intensity of } \\
\text { illumination (lux) }\end{array}$} & \multirow[b]{2}{*}{$\sigma\left(\Omega \mathrm{cm}^{-1}\right)\left(10^{-5}\right)$} & \multirow[b]{2}{*}{$N_{\text {eff }}\left(\mathrm{cm}^{-3}\right)\left(10^{10}\right)$} & \multirow[b]{2}{*}{$S\left(\mathrm{~s}^{-1}\right)\left(10^{6}\right)$} & \multicolumn{2}{|c|}{$E(\mathrm{eV})$} \\
\hline & & & & & $E_{1}$ & $E_{2}$ \\
\hline \multirow[t]{5}{*}{303} & 40,000 & $2 \cdot 28$ & $24 \cdot 58$ & $14 \cdot 69$ & 0.43 & 0.45 \\
\hline & 60,000 & $2 \cdot 84$ & $30 \cdot 66$ & $18 \cdot 33$ & 0.44 & 0.46 \\
\hline & 80,000 & $3 \cdot 27$ & $35 \cdot 30$ & $21 \cdot 11$ & 0.45 & 0.47 \\
\hline & 100,000 & $4 \cdot 19$ & $45 \cdot 18$ & $27 \cdot 01$ & 0.45 & 0.48 \\
\hline & 120,000 & $5 \cdot 03$ & $54 \cdot 24$ & $32 \cdot 43$ & 0.46 & 0.48 \\
\hline \multirow[t]{5}{*}{333} & 40,000 & $4 \cdot 19$ & $45 \cdot 15$ & $28 \cdot 86$ & $0 \cdot 51$ & $0 \cdot 54$ \\
\hline & 60,000 & $5 \cdot 19$ & $55 \cdot 93$ & $35 \cdot 76$ & 0.52 & $0 \cdot 54$ \\
\hline & 80,000 & $6 \cdot 70$ & $72 \cdot 26$ & $46 \cdot 20$ & 0.53 & $0 \cdot 55$ \\
\hline & 100,000 & $8 \cdot 38$ & $90 \cdot 32$ & 57.75 & 0.55 & 0.56 \\
\hline & 120,000 & $10 \cdot 35$ & 111.53 & $71 \cdot 31$ & $0 \cdot 55$ & 0.57 \\
\hline \multirow[t]{5}{*}{363} & 40,000 & $5 \cdot 94$ & $64 \cdot 08$ & $42 \cdot 88$ & $0 \cdot 63$ & $0 \cdot 66$ \\
\hline & 60,000 & $7 \cdot 72$ & $83 \cdot 18$ & $55 \cdot 67$ & $0 \cdot 64$ & 0.67 \\
\hline & 80,000 & $10 \cdot 29$ & $110 \cdot 80$ & $74 \cdot 15$ & 0.65 & 0.68 \\
\hline & 100,000 & $12 \cdot 48$ & $134 \cdot 48$ & $90 \cdot 00$ & $0 \cdot 66$ & 0.69 \\
\hline & 120,000 & $14 \cdot 62$ & $157 \cdot 53$ & $105 \cdot 4$ & $0 \cdot 67$ & $0 \cdot 70$ \\
\hline
\end{tabular}

$$
S_{\mathrm{t}} \approx 10^{-10} / \varepsilon^{2} \mathrm{~cm}^{2} .
$$

Using the relation, $S=N_{\text {eff }} v_{\text {th }} S_{\mathrm{t}}$, the frequency factor, $S$, at different ambient conditions were evaluated. The different calculated values of $N_{\text {eff }}$ and $S$ are shown in table 1.

Table 1. Calculated values of photoconductivity $(\sigma)$, effective density of states $\left(N_{\text {eff }}\right)$, frequency factor $(S)$ and trap depth $(E)$ of a CdSe thin film $\left(t_{\mathrm{t}}=2000 \AA, T_{\mathrm{s}}=473 \mathrm{~K}\right)$ illuminated by white light of different intensities and at different ambient temperatures $(T)$. 
From the table, it is seen that there is a gradual increase in the value of $S$ with ambient temperature as well as with intensity of illumination. Using values of $p$, evaluated from the slopes of $\ln \left(I_{0} / I_{\mathrm{t}}\right)$ vs time, $t$ plots, under different conditions, shown in figure $1(\mathrm{a}, \mathrm{b}, \mathrm{c})$, and $S$, in relation (3), the trap depths at different ambient conditions were calculated. The different values of trap depths are also systematically presented in table 1 . From figure $1(a, b, c)$, it is observed that there exist two different
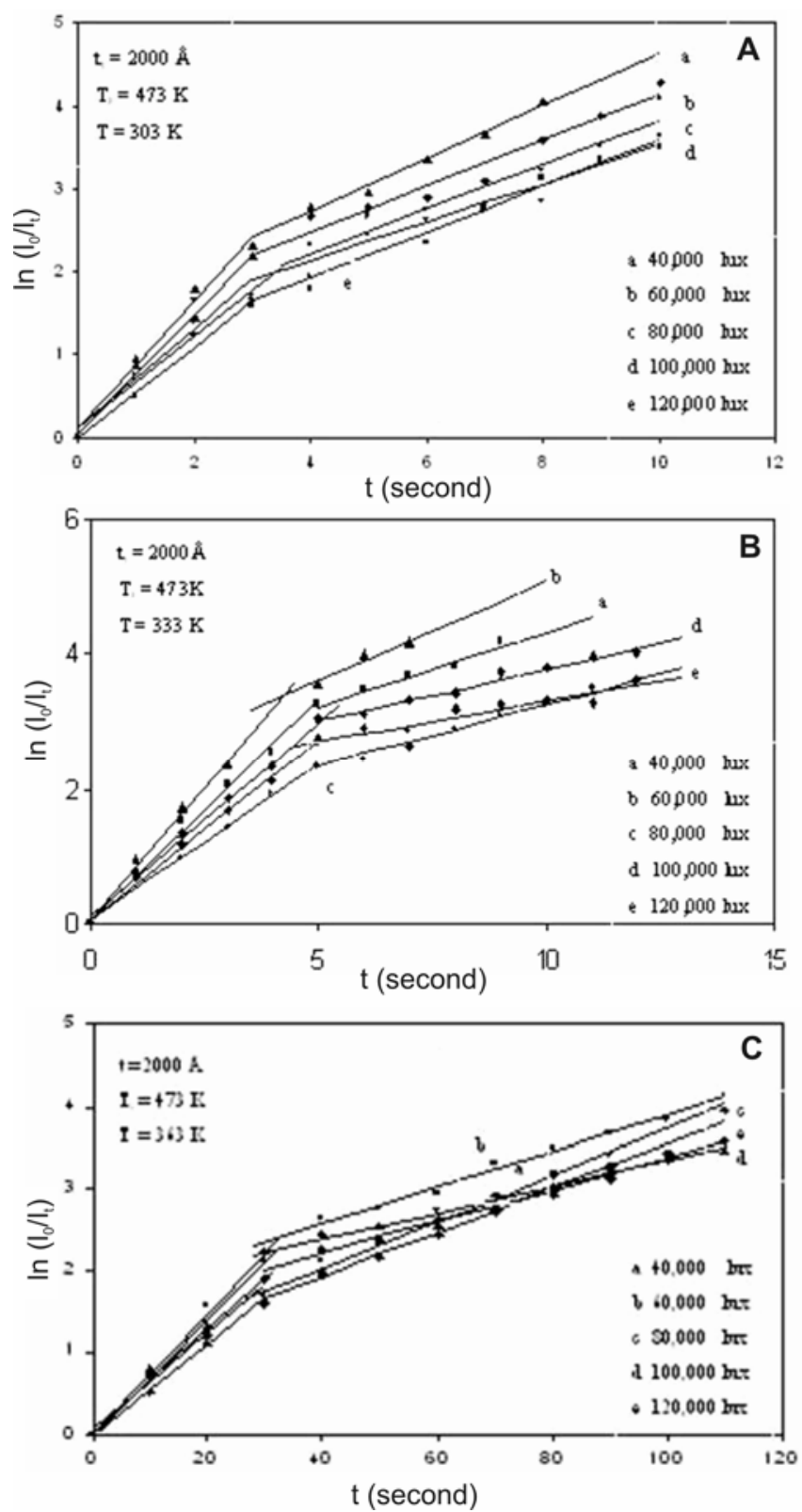

Figure 1. (A) $\ln \left(I_{0} / I_{\mathrm{t}}\right)$ vs time, $t$, plot of a CdSe thin film grown at elevated $T_{\mathrm{s}}$ and illuminated by white light, at an ambient temperature, $T=303 \mathrm{~K},(\mathbf{B}) \ln \left(I_{0} / I_{\mathrm{t}}\right)$ vs time, $t$, plot of a CdSe thin film grown at elevated $T_{\mathrm{s}}$ and illuminated by white light, at an ambient temperature $T=333 \mathrm{~K}$ and $(\mathbf{C}) \ln \left(I_{0} / I_{\mathrm{t}}\right)$ vs time $t$, plot of a CdSe thin film grown at elevated $T_{\mathrm{s}}$ and illuminated by white light, at an ambient temperature $T=363 \mathrm{~K}$. slopes in $\ln \left(I_{0} / I_{\mathrm{t}}\right)$ vs time $t$ plots, which indicates the existence of two distinct trap levels, $E_{1}$ and $E_{2}$, at any ambient temperature. It may be noted that photocurrent in these thin films was found to obey a sub-linear relation with the intensity of illumination which could be explained on the basis of defect controlled photoconductivity mechanism.

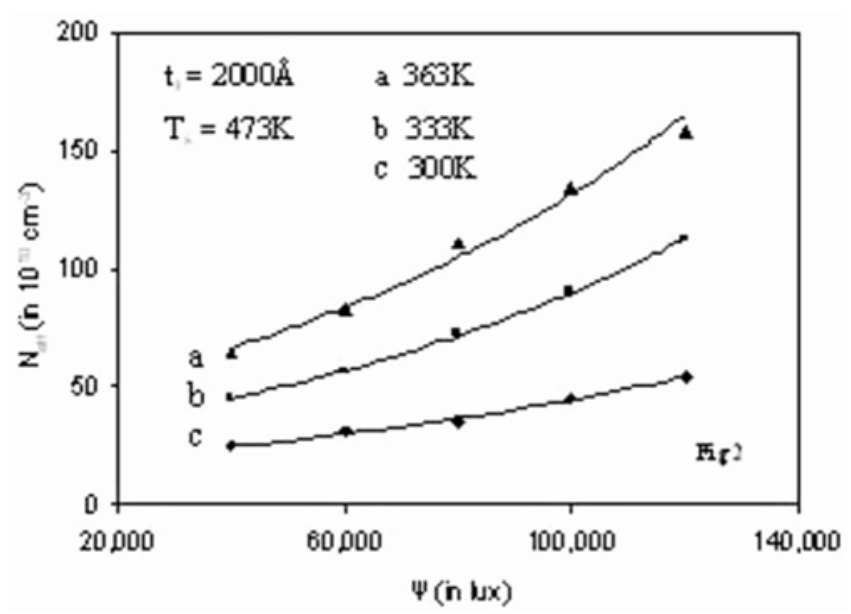

Figure 2. Density of states $\left(N_{\text {eff }}\right)$ vs intensity of illumination $(\psi)$ of a CdSe thin film grown at elevated $T_{\mathrm{s}}$ at different ambient temperatures $(T)$.
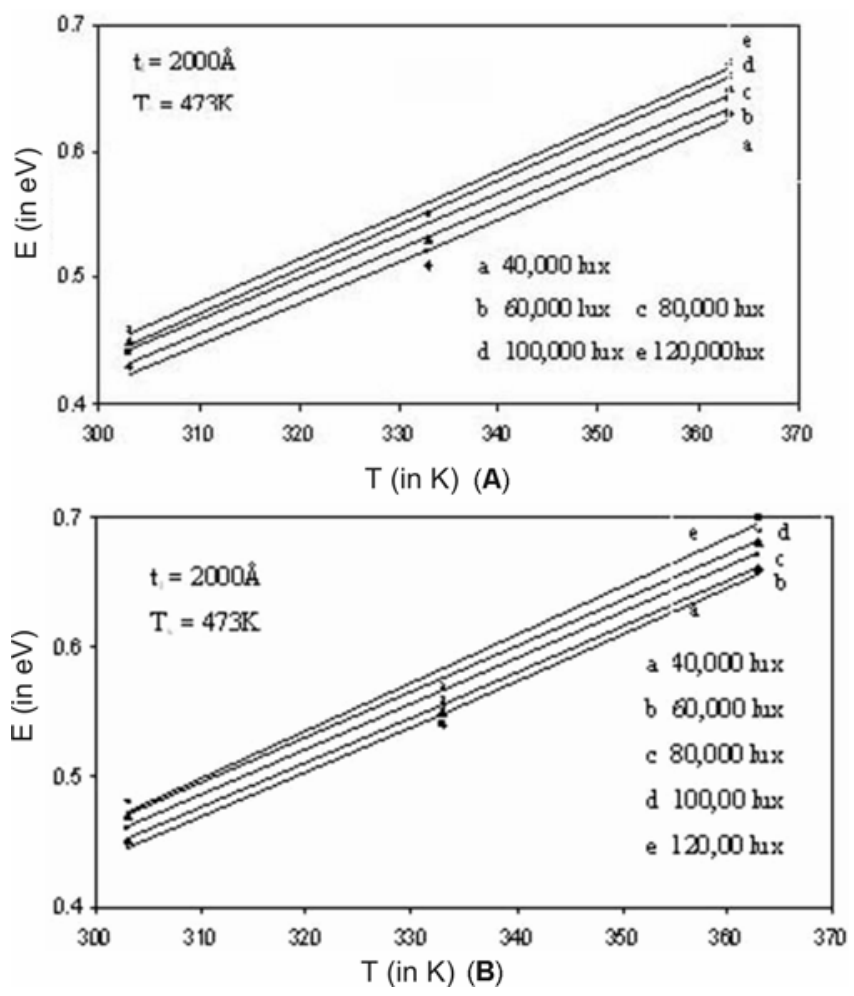

Figure 3. (A) Plot of trap depth $\left(E_{1}\right)$ vs ambient temperature $(T)$ at different intensity of illuminations of a CdSe thin film grown at elevated $T_{\mathrm{s}}$ and $(\mathbf{B})$ plot of trap depth $\left(E_{2}\right)$ vs ambient temperature $(T)$ at different intensity of illuminations of a CdSe thin film grown at elevated $T_{\mathrm{s}}$. 
Thin films of CdSe, grown at elevated $T_{\mathrm{s}}$, are found to be of polycrystalline nature having grains of different sizes. These films have some built in potential barriers, $\phi_{\mathrm{b}}$, at the grain boundaries and this grain boundary potential can be expressed as (Sarmah and Das 1991)

$$
\Phi_{\mathrm{b}}=\left(Q_{\mathrm{t}}-\Delta P_{\mathrm{t}}\right) / 8 \varepsilon \varepsilon_{\mathrm{o}} n,
$$

where $n$ is the photogenerated carrier density, which is greater than the density of majority carrier traps, $Q_{\mathrm{t}}$, localized at the grain boundaries, $\varepsilon$ and $\varepsilon_{\mathrm{o}}$ are the dielectric constants of the material of thin film and free space, respectively. $\Delta P_{\mathrm{t}}$ is the density of trapped minority carriers under illumination.

Due to the absorption of illuminating radiation $(\psi)$, free electron hole pairs are generated. The photogenerated electrons and holes which are in excess to the thermal equilibrium number density of carriers are responsible for photoconduction process. One part of the photogenerated carriers recombine with respective opposite charges localized at grain boundary depletion regions, thereby reducing the grain boundary potential barriers, $\phi_{\mathrm{b}}$ and the rest take part in the photoconduction process. As a result of reduction of $\phi_{\mathrm{b}}$ the effective mobility of the carriers increases. This process is known as barrier modulation. The effective mobility, $\mu^{*}$, is given by the relation (Petritz 1956)

$$
\mu^{*}=\mu_{\mathrm{o}} \exp \left(-q \Phi_{\mathrm{b}} / k T_{\mathrm{o}}\right)
$$

where $T_{\mathrm{o}}$ is the characteristics temperature of the film system, $\mu_{\mathrm{o}}$ the mobility of the carrier with no barrier effect. Due to the increase in $\mu^{*}$ also, the conductivity of the sample increases.

Thus the increase in photoconductivity is due to two contributors viz. one from the resultant increase in the photogenerated carriers and the other from the increase in the effective mobility. Thus the photoconductivity is given by

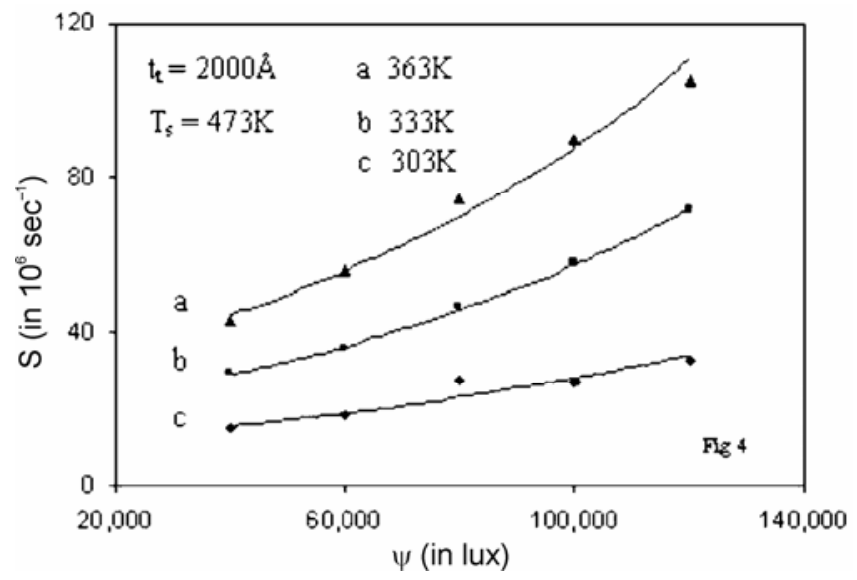

Figure 4. Frequency factor $(S)$ vs intensity of illumination $(\psi)$ of a CdSe thin film grown at elevated $T_{\mathrm{s}}$ at different ambient temperatures.

$$
\sigma_{\mathrm{ph}}=N_{\text {eff }} q \mu^{*},
$$

where $N_{\text {eff }}$ is the effective density of states in the conduction band under illumination which is assumed to be the numbers of occupied energy levels $n$, in the conduction band by the electrons. Thus $N_{\text {eff }}$ can be written as

$$
N_{\text {eff }}=2\left(2 \pi k T / h^{2}\right)^{3 / 2}\left(m_{\mathrm{e}} * m_{\mathrm{h}} *\right)^{3 / 4} \exp \left(-E_{\mathrm{g}} / k T\right) \text {. }
$$

Therefore

$$
\sigma_{\mathrm{ph}}=C \exp \left(-q \phi_{\mathrm{b}} / k T_{\mathrm{o}}\right)
$$

where

$$
C=2\left(2 \pi k T / h^{2}\right)^{3 / 2}\left(m_{\mathrm{e}}^{*} m_{\mathrm{h}}\right)^{3 / 4} \exp \left(-E_{\mathrm{g}} / k T\right) q \mu_{\mathrm{o}} .
$$

From (12) one can write

$$
\ln \sigma_{\mathrm{ph}}=\ln C+\left(-q \Phi_{\mathrm{b}} / k T_{\mathrm{o}}\right) \text {. }
$$

It can be seen from figure 2 that the values of $N_{\text {eff }}$ $\left(N_{\text {eff }}=n\right)$ calculated from experimental $\sigma_{\mathrm{ph}}$ data bear an exponential relation with the incident intensity of illumination, $\psi$. This is in good agreement with (12). It may be noted that $\ln N_{\text {eff }} \propto \psi$. This implies that $\phi_{\mathrm{b}}$ is inversely
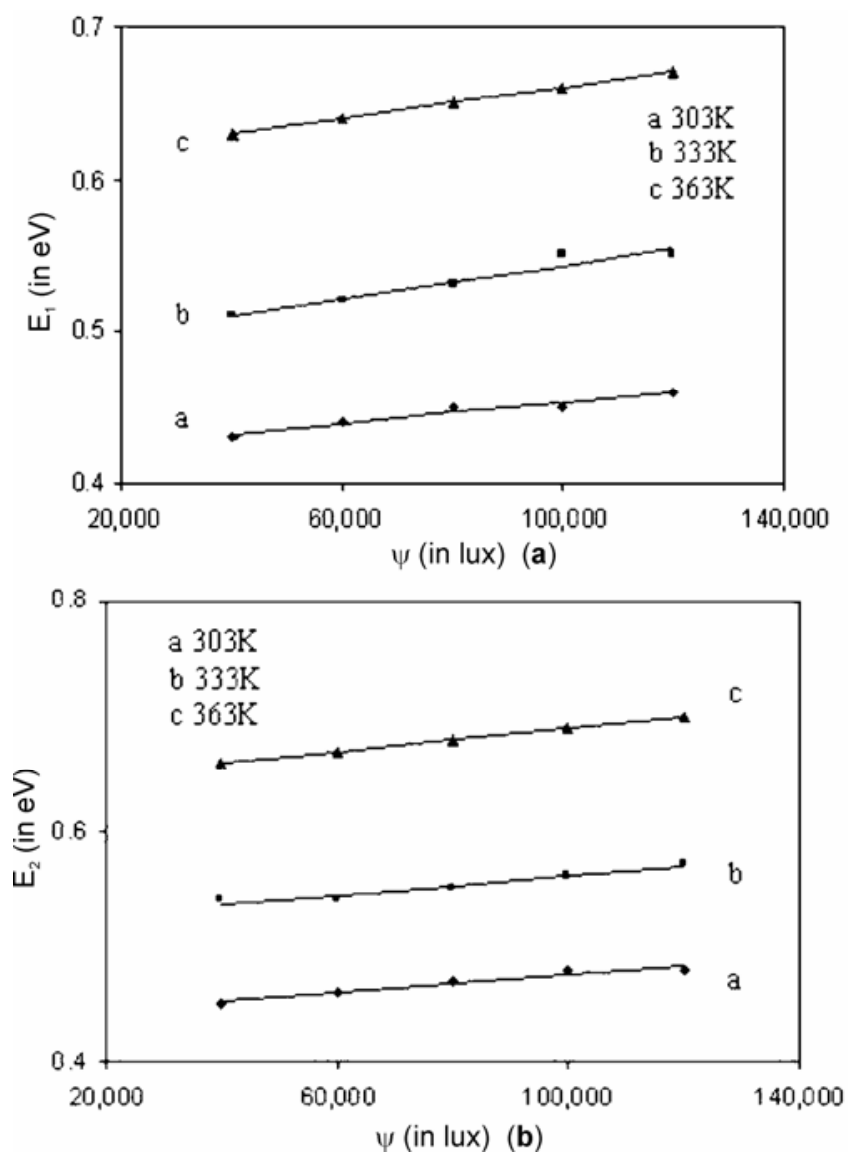

Figure 5. (a) Trap depth $\left(E_{1}\right)$ vs intensity of illumination $(\psi)$ at different ambient temperatures of a CdSe thin film grown at elevated $T_{\mathrm{s}}=473 \mathrm{~K}$ and $(\mathbf{b})$ trap depth $\left(E_{2}\right)$ vs intensity of illumination $(\psi)$ at different ambient temperatures of a CdSe thin film grown at elevated $T_{\mathrm{s}}=473 \mathrm{~K}$. 
proportional to $\psi$, which justifies the barrier modulation process.

The depth $E$, which a trap is required to possess in order that an electron may be trapped in it, varies with depth of trap. Hence the number of traps in which an electron spends a mean time, $t$, will vary with temperature and therefore, $I_{\mathrm{t}}$ varies with temperature. Figures 3(a) and (b) show the variation of trap energy with temperature. It is clear from the figures that trap depths $E_{1}$ and $E_{2}$ are not single valued but varies over a range with temperature.

The traps may be emptied by the absorption of optical radiation of different intensity as well as by the utilization of thermal energy. So the trap depths can be calculated from the spectrum of photoconductivity decay curve (Bube 1967). As already mentioned, $S=N_{\text {eff }} v_{\text {th }} S_{\mathrm{t}}$, where $N_{\text {eff }}$ is the density of states, $v_{\text {th }}$ the thermal velocity of the electrons, $S_{\mathrm{t}}$ the capture cross section of the electron at any trap level. With the increase in intensity of illumination, $N_{\text {eff }}$ increases exponentially (figure 2) whereas $v_{\text {th }}$ and $S_{\mathrm{t}}$ remain constant. So the frequency factor, $S$, also increases exponentially with illumination as shown in figure 4 . It may be noted that $\ln S \propto \psi$, hence

$$
\ln S=C \psi,
$$

where $C$ is the proportionality constant. So, $S$, which is directly proportional to $N_{\text {eff }}$ (or $n$ ), becomes the main contributory term of the trap energy. The ratio $I_{0} / I_{\mathrm{t}}$ is nearly constant at any illumination level. Thus the term $\ln \left\{\ln \left(I_{0} / I_{\mathrm{t}}\right) / t\right\}$ in (3) has a little contribution in the trap energy. Hence the dependence of trap energy, $E$, is basically determined by the term $\ln S$. Therefore, $E$ is expected to be proportional to $\psi$. This is seen to be justified by the plots $E_{1}$ vs $\psi$ and $E_{2}$ vs $\psi$ as shown in figures 5(a) and (b), respectively.

\section{Conclusions}

Photoconductivity of thermally deposited CdSe thin films is mainly because of two factors one due to increase of photogenerated carriers, known as carrier modulation and other due to increase of effective mobility, known as barrier modulation. In these films trapping centres are responsible for controlling the photocurrent. Both shallow and deep traps are found to be available in these films. The observed trap depths are not single valued and there is a quasi-continuous distribution of various traps.

\section{References}

Al-Ani S K J, Mohammed H H and Al-Fwade E M N 2002 Renew. Energ. 25585

Baban C, Rusu G G, Nicolaescu I I and Rusu G I 2000 J. Phys. Condens. Matter. 127687

Baban C, Rusu G I and Prepelita P 2005 J. Optoelectron. Adv. Mater. 7817

Bube R H 1960 Photoconductivity of solids (New York: Wiley) pp 61, 278

Bube R H 1967 Physics and chemistry of II-VI compounds (eds) M Aven and J S Prener (Amsterdam: North Holland Publishing Company) pp 660, 681

Devlin S S 1967 Physics and chemistry of II-VI compounds (eds) M Aven and J S Prener (Amsterdam: North Holland Publishing Company) pp 587, 589

Kale R B and Lokhande C D 2005 Semicond. Sci. Technol. 201

Kalita P K, Sarma B K and Das H L 1999 Indian J. Pure \& Appl. Phys. 37885

Kalita P K, Sarma B K and Das H L 2003 Bull. Mater. Sci. 26 613

Kindleysides L and Woods J 1970 J. Phys. D: Appl. Phys. 3 451

Oduor A O and Gould R D 1995 Thin Solid Films 270387

Pal U, Samanta D, Ghorai S and Chaudhuri A K 1993 J. Appl. Phys. 746368

Petritz R L 1956 Phys. Rev. 1041508

Randall J T and Wilkins M H F 1945 Proc. R. Soc. (London) A184 366

Sarmah K C and Das H L 1991 Thin Solid Films 19829

Sathyalatha K C, Uthanna S and Jayaramareddy P 1989 Thin Solid Films 174233

Serpi A 1976 J. Phys. D: Appl. Phys. 91881

Shreekanthan K N, Rajendra B V, Kasturi V B and Shivakumar G K 2003 Cryst. Res. Technol. 3830

Srivastava B N and Singh S 1970 Indian J. Pure \& Appl. Phys. 8721 